\title{
IgG4-related pachymeningitis masquerading as foramen magnum meningioma: illustrative case
}

\author{
Haggai Suisa, MD, ${ }^{1}$ Jean Francois Soustiel, MD, ${ }^{1,2}$ and Yuval Grober, MD ${ }^{1}$ \\ ${ }^{1}$ Department of Neurosurgery, Galilee Medical Center, Nahariya, Israel; and ${ }^{2}$ The Azrieli Faculty of Medicine, Bar-llan University, Safed, Israel
}

BACKGROUND Immunoglobulin G4-related disease (IgG4-RD) is an immune-mediated inflammatory condition with potential multiorgan involvement. Common manifestations include autoimmune pancreatitis and retroperitoneal fibrosis. Pathological analysis reveals lymphoplasmacytic infiltrate rich in lgG4-positive cells and characteristic storiform fibrosis. Early treatment with glucocorticoids may prevent progression to poorly responsive fibrotic disease.

OBSERVATIONS A 63-year-old female patient presented with reports of left-sided headaches, nausea, and photophobia in addition to recently diagnosed chronic rhinosinusitis (CRS). Neurological examination revealed dysarthria secondary to left hypoglossal nerve palsy. Computed tomography (CT) revealed a contrast-enhancing extraaxial mass at the left craniocervical junction, CRS with secondary hyperostotic reaction, and multiple hypodense lesions involving the occipital bone. Magnetic resonance imaging revealed a dural-based lesion involving the foramen magnum and invading the left hypoglossal canal. The patient underwent a far-lateral craniotomy. Histopathological analysis revealed severe lymphoplasmacytic inflammation, storiform fibrosis and rich plasma-cell population positive for lgG4. Serum lgG4 was markedly elevated. Total-body CT showed no systemic involvement. The patient was diagnosed with lgG4-RD and was prescribed prednisone, with normalization of her lgG4 levels after 1 month.

LESSONS IgG4-RD may mimic a variety of diseases, including skull-base meningiomas and CRS. Accurate diagnosis and expedited administration of steroids may prevent unnecessary interventions and progression to treatment-resistant fibrosis.

https://thejns.org/doi/abs/10.3171/CASE21398

KEYWORDS IgG4-RD; IgG4 related disease; foramen magnum meningioma; CRS; chronic rhinosinusitis; hypoglossal palsy

Immunoglobulin G4-related disease (IgG4-RD) is an immune-mediated inflammatory condition with potential multiorgan involvement. ${ }^{1}$ Examples of common manifestations include autoimmune pancreatitis, sclerosing cholangitis, sclerosing sialadenitis (also known as Mikulicz disease), and retroperitoneal fibrosis. ${ }^{2}$ Patients may present with a slow-growing mass or diffuse enlargement of a specific organ. Seventy-five percent of patients suffer from multiorgan involvement. ${ }^{3}$ Although many patients are asymptomatic at the time of diagnosis, common signs and symptoms include lymphadenopathy, asthma or allergy-like symptoms, and weight loss. ${ }^{4}$ Pathologic analysis of involved organs reveals a lymphoplasmacytic infiltrate enriched in lgG4-positive plasma cells, a characteristic storiform fibrosis, obliterative phlebitis, and modest tissue eosinophilia. ${ }^{5}$ Elevated serum lgG4 levels are recognized in approximately two-thirds of patients. ${ }^{6}$ Growing evidence suggests an autoimmune basis for the disease, mediated mainly by CD4+ and T-follicular helper cells. Surprisingly, IgG4 antibodies themselves are not pathogenic or specific and may actually play an antiinflammatory role in this disease. ${ }^{7}$ The most prevalent cells within lgG4-RD affected tissues are a clonally expanded population of CD4+ cytotoxic T lymphocytes. These cells produce important fibrosis-promoting mediators, such as interleukin-1 and transforming growth factor-beta. ${ }^{8}$

The prevalence of IgG4-RD is thought to be largely underestimated worldwide, mainly because of the lack of internationally accepted diagnostic criteria and clinical awareness. In a large cohort study that included almost 500 patients, the mean age at diagnosis was $59{ }^{9}$ Although the predilection for men mainly includes manifestations such as autoimmune pancreatitis, tubulointerstitial nephritis, and retroperitoneal fibrosis, manifestations of the head and neck, such as sialadenitis, are

ABBREVIATIONS CRS = chronic rhinosinusitis; CT = computed tomography; IgG4-RD = immunoglobulin G4-related disease; MRI = magnetic resonance imaging INCLUDE WHEN CITING Published December 6, 2021; DOI: 10.3171/CASE21398.

SUBMITTED July 7, 2021. ACCEPTED July 12, 2021.

(C) 2021 The authors, CC BY-NC-ND 4.0 (http://creativecommons.org/licenses/by-nc-nd/4.0/). 
more common in women. ${ }^{10}$ Although histopathology with lgG4 staining is considered the mainstay of lgG4-RD diagnosis, it is not diagnostic by itself. ${ }^{11}$ Elevated lgG4 levels and C3 and C4 hypocomplementemia are important aids in the diagnosis but are also not diagnostic alone because of lack of sufficient specificity and sensitivity. These markers can also be used to confirm and follow glucocorticoid response in patients. Early treatment with glucocorticoids may delay or even prevent the progression from treatment-responsive inflammatory disease to poorly responsive fibrotic disease with end-organ damage. However, many patients eventually require additional treatment modalities, such as the anti-CD20 monoclonal antibody rituximab. ${ }^{11}$

\section{Illustrative Case}

A 63-year-old female patient of Bedouin descent presented to the neurosurgical outpatient clinic with reports of episodic left-sided headaches accompanied by nausea and photophobia. Her attacks usually last a few hours, although some may persist for up to 24 hours. Medical history was positive for hypertension, obesity, chronic ischemic heart disease, chronic obstructive pulmonary disease, controlled type 2 diabetes mellitus, and hyperlipidemia. In addition, the patient was recently diagnosed with chronic sinusitis, with persistent reports of thick nasal discharge and chronic congestion, reduced sense of smell, and constant vague pain around her eyes and forehead. Her physical examination was unremarkable, apart from frontal and maxillary sinus tenderness to palpation. Neurological examination revealed mild dysarthria and left-sided deviation of her tongue on protrusion, suggesting possible palsy of cranial nerve XII. Lab results showed mild eosinophilia (7.8\%) and normocytic anemia. Formal ear/nose/throat evaluation with fiberoptic endoscopy was negative for additional lower cranial nerve involvement. Head and upper cervical spine computed tomography (CT) revealed a 3-cm hyperdense mass at the left craniocervical junction engulfing the medulla oblongata. The mass underwent avid contrast enhancement (Fig. 1). In addition, the maxillary, ethmoidal, and frontal sinuses showed extensive opacification with secondary hyperostotic reaction and intrasinus calcifications (Fig. 2A) suggestive of chronic sinusitis. Finally, her occipital bone contained numerous hypodense oval lesions of unknown etiology (Fig. 2B).

Magnetic resonance imaging (MRI) revealed a contrast-enhancing dural-based lesion involving the left margin of the foramen magnum and extending cranially toward the clivus and dorsum sella
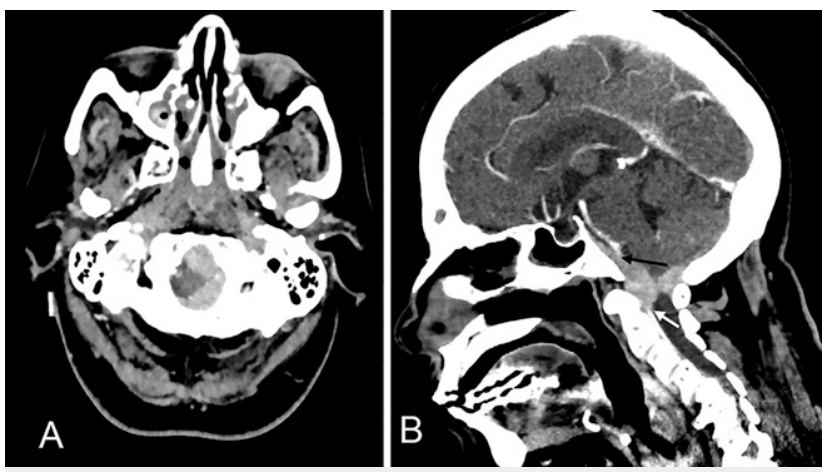

FIG. 1. Axial (A) and sagittal (B) contrast-enhanced CT scan showing an avidly enhancing extraaxial mass in the left craniocervical junction. The mass has an apparent dural tail that extends to the midclivus (white arrow) and the odontoid base (white arrow). It engulfs the left anterolateral and posterolateral medulla.
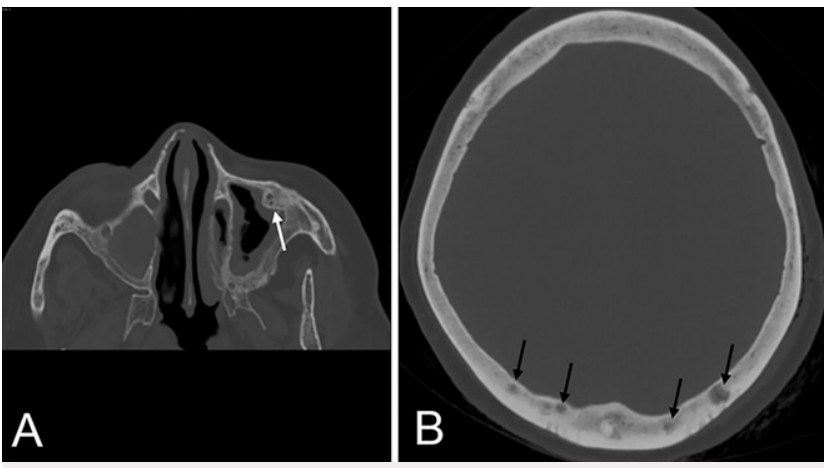

FIG. 2. Axial CT, bone window. A: Complete opacification of the right maxillary sinus. The left maxillary sinus is partially opacified, with secondary hyperostosis (white arrow). B: Several oval hypodense lesions in the patient's occipital bone (black arrows).

(Fig. 3A), with mass effect on the posterolateral and anterolateral medulla (Fig. 3B). The lesion appeared to invade the left hypoglossal foramen and canal (Fig. 3C). Diffusion-weighted MRI showed markedly reduced diffusion (Fig. 4A), which is not typical of lowgrade meningiomas. Fluid-attenuated inversion recovery MRI showed central hypointensity with a thin hyperintense rim (Fig. 4C), also an atypical finding for a meningioma. A presumed diagnosis of a foramen magnum meningioma followed, and the patient underwent an elective craniotomy using the far-lateral approach, with close lower cranial nerve monitoring. During surgery, the patient's atlanto-occipital membrane appeared dense and fibrotic. After drilling of the posteromedial occipital condyle, the dura was elevated and the lesion was removed up to the lower clivus. Macroscopically, extensive dural involvement and nonspecific bony changes were consistent with en plaque meningioma. The clival portion of the lesion appeared thin and showed no mass effect on the adjacent brainstem. Given the necessary retraction and possible risk to critical structures, mainly the vagus nerve, the clival component was left in place. The following day, the patient showed complete recovery and was ambulating freely in the department. No new neurological deficits were documented. Histopathological assessment of the fibrous atlanto-occipital membrane and the dural lesion revealed a fibrous connective tissue with thick collagen fibers arranged in a storiform pattern (Fig. 5A and B). The tissue demonstrated marked lymphocytic inflammation, with numerous plasma cells positive for CD138 (Fig. 5C), kappa and lambda light chains. Most plasma cells were positive for $\operatorname{lgG}$, and $40 \%$ of these stained positive for $\lg \mathrm{G} 4$ (Fig. 5D).

The presumed diagnosis of meningioma was replaced with probable IgG4-related disease. The patient's serum was subsequently tested for $\lg G 4$, total $\lg G, \lg M, \lg A, \lg E, C 3$, and C4. All levels were normal, except for marked elevation of the patient's lgG4 levels (348 $\mathrm{mg} / \mathrm{dL}$ ), which was almost three times the laboratory's upper reference limit of $121 \mathrm{mg} / \mathrm{dL}$. A definitive diagnosis of IgG4-RD was made, and the patient underwent a contrast-enhanced total-body CT scan to rule out any systemic involvement. Her whole-body CT was negative for any involvement outside the head and neck area. The patient was started on $60 \mathrm{mg}$ of prednisone with calcium and vitamin D supplementation, prophylactic oral trimethoprim-sulfamethoxazole, and frequent monitoring of blood glucose levels, with appropriate adjustment of her antidiabetic regimen. However, because of uncontrolled diabetes despite 


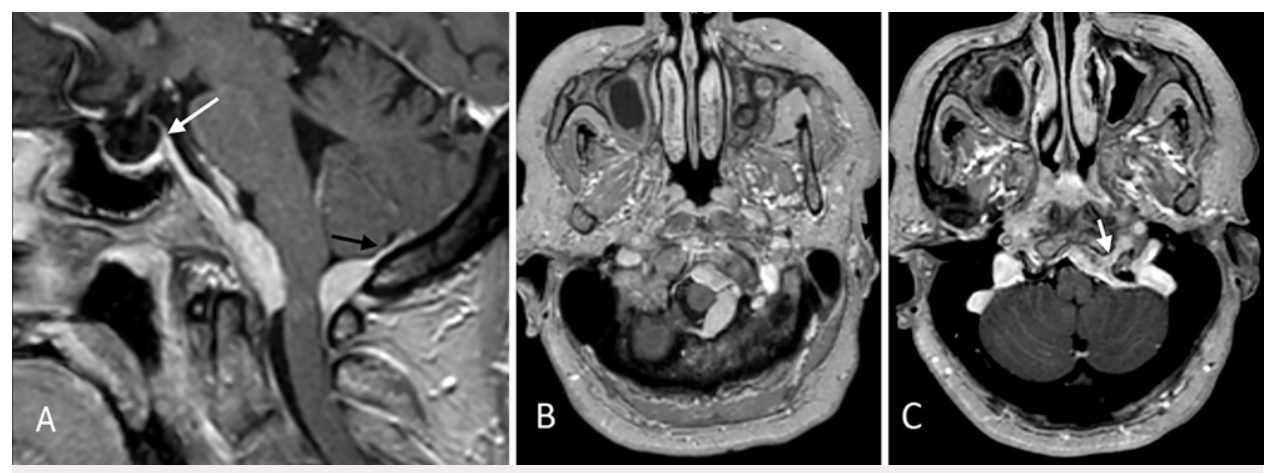

FIG. 3. Gadolinium-enhanced T1-weighted MRI. A: Midsagittal section shows a homogeneously enhancing extraaxial mass engulfing the medulla. The mass has a clear dural tail extending toward the dorsum sella (white arrow) and internal occipital crest (black arrow). B: Axial section at the level of the foramen magnum showing mass effect on the posterolateral and anterolateral medulla. C: Axial section at the hypoglossal canal level showing invasion into the left hypoglossal canal (white arrow).

numerous treatment adjustments, she was switched to intravenous rituximab (1 g every 15 days). A follow-up serum test after 1 month revealed normalization of her lgG4 levels.

\section{Discussion}

\section{Observations}

Intracranial involvement in IgG4-RD has become better recognized only recently. The most common central nervous system manifestations of IgG4-RD include hypophysitis and pachymeningitis. ${ }^{12}$ lgG4-related pachymeningitis involves inflammation with diffuse or focal thickening of the meninges (usually the dura mater). ${ }^{13}$ Dural lesions demonstrate homogeneous contrast enhancement on both MRI and CT scans. ${ }^{14}$ Accurate diagnosis and rapid administration of glucocorticoids are essential in preventing irreversible fibrosis. The rarity of cranial base pseudotumors in IgG4-RD has limited the characterization of this entity to case reports. ${ }^{15,16}$ In a recently published multicenter review of all IgG4-RD cases in three tertiary care referral centers in the United States and Italy between 2003 and $2019,{ }^{15} 11$ cases of skull base involvement were documented. The median age at diagnosis was 58 years. Ninety-one percent of patients had solitary lesions, and only $9 \%$ suffered from systemic disease. The most common reports at presentation were headaches and diplopia. The most common neurological signs were abducens nerve palsy (55\%) followed by facial nerve palsy (18\%). Six of 11 cases involved the cavernous sinus and two of 11 involved the petroclival ridge. To our knowledge, this is the second published case
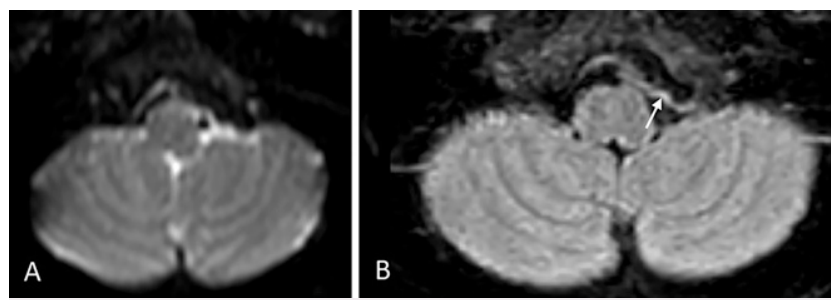

FIG. 4. A: Diffusion-weighted imaging MRI shows markedly restricted diffusion within the lesion. B: Fluid-attenuated inversion recovery MRI shows a central hypointensity with a thin hyperintense rim (white arrow). Both findings are highly atypical for meningiomas. of an IgG4-related pseudotumor mimicking a foramen magnum meningioma. The first report in 2015 by Okano et al. ${ }^{16}$ presented a patient with a right paraclinoidal and a left foramen magnum lesion. This patient suffered from an lgG4-related autoimmune pancreatitis several years before his current presentation. This makes our case the first isolated IgG4-related foramen magnum pseudotumor in literature. Of note, isolated hypoglossal nerve palsy is rare in everyday clinical practice, with a limited differential diagnosis. ${ }^{17}$ Chronic rhinosinusitis (CRS) was first identified in 2011 as one of the extrapancreatic manifestations of IgG4-RD by Moteki et al. ${ }^{18} \mathrm{CRS}$ is suspected in patients presenting with persistent symptoms of nasal congestion, facial pain, and possibly anosmia. ${ }^{19}$ However, IgG4-related CRS differs from regular CRS by its longer clinical course, which may explain the marked hyperostotic reaction seen in our patient. While reports of skull-base lytic lesions in lgG4 patients
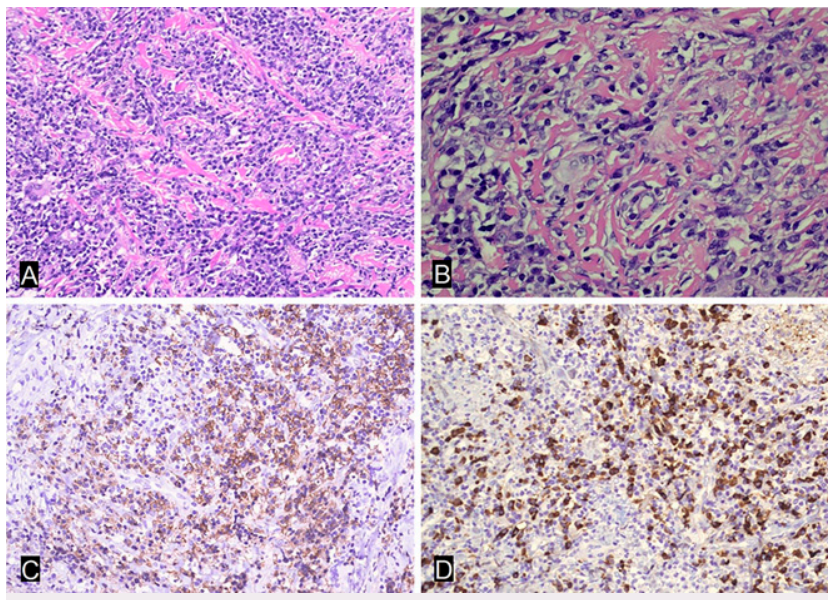

FIG. 5. Histopathological analysis. A: Hematoxylin and eosin stain demonstrates dense collagenous tissue with hypereosinophilic collagenous bands and a rich lymphoplasmacytic infiltrate. Original magnification $\times 200$. B: Hematoxylin and eosin stain demonstrates typical concentric (storiform) fibrosis. Original magnification $\times 400$. C: Immunostaining for CD138 shows a rich plasma cell component. Original magnification $\times 200$. D: IgG4 immunostaining shows lgG4-positive plasma cell population. Original magnification $\times 200$. 
are rare, reports of calvarial involvement are exceedingly rare. In a 2021 systematic review of more than 1,352 cases of IgG4-RD, ${ }^{20}$ 357 cases included head and neck involvement. Of these, only a handful of cases described calvarial involvement, mainly of the temporal bone (eight cases). To the best of our knowledge, this is the first presentation that combines pachymeningeal inflammation with CRS and calvarial lesions.

\section{Lessons}

This article combines a rare neurological presentation (isolated hypoglossal nerve palsy and a unique clinical entity) of IgG-RD pseudotumor mimicking a foramen magnum meningioma with lgG4related CRS and hypodense calvarial lesions. We believe that in combination with the imaging and histopathological analysis, this case may assist clinicians in avoiding common pitfalls related to the diagnosis of $\lg G 4-R D$, possibly leading to accelerated diagnosis and treatment, which may prevent unnecessary intervention and progression to treatment-resistant fibrosis.

\section{References}

1. Stone JH, Zen Y, Deshpande V. IgG4-related disease. N Engl J Med. 2012;366(6):539-551.

2. Khosroshahi A, Stone JH. A clinical overview of IgG4-related systemic disease. Curr Opin Rheumatol. 2011;23(1):57-66.

3. Okazaki K, Uchida K, Koyabu M, Miyoshi H, Takaoka M. Recent advances in the concept and diagnosis of autoimmune pancreatitis and IgG4-related disease. J Gastroenterol. 2011;46(3):277-288.

4. Cheuk W, Chan JK. IgG4-related sclerosing disease: a critical appraisal of an evolving clinicopathologic entity. Adv Anat Pathol. 2010;17(5):303-332.

5. Deshpande V, Zen Y, Chan JK, et al. Consensus statement on the pathology of IgG4-related disease. Mod Pathol. 2012;25(9):1181-1192.

6. Carruthers MN, Khosroshahi A, Augustin T, Deshpande V, Stone $\mathrm{JH}$. The diagnostic utility of serum lgG4 concentrations in IgG4related disease. Ann Rheum Dis. 2015;74(1):14-18.

7. Mahajan VS, Mattoo H, Deshpande V, Pillai SS, Stone JH. IgG4related disease. Annu Rev Pathol. 2014;9:315-347.

8. Mattoo H, Mahajan VS, Maehara T, et al. Clonal expansion of $\mathrm{CD} 4(+)$ cytotoxic T lymphocytes in patients with lgG4-related disease. J Allergy Clin Immunol. 2016;138(3):825-838.

9. Wallace ZS, Zhang Y, Perugino CA, Naden R, Choi HK, Stone JH. Clinical phenotypes of lgG4-related disease: an analysis of two international cross-sectional cohorts. Ann Rheum Dis. 2019;78(3):406-412.

10. Wang $L$, Zhang $P$, Zhang $X$, et al. Sex disparities in clinical characteristics and prognosis of immunoglobulin G4-related disease: a prospective study of 403 patients. Rheumatology (Oxford). 2019; 58(5):820-830.

11. Khosroshahi A, Wallace ZS, Crowe JL, et al. International consensus guidance statement on the management and treatment of IgG4-related disease. Arthritis Rheumatol. 2015;67(7):1688-1699.

12. Joshi $D$, Jager $R$, Hurel $S$, et al. Cerebral involvement in lgG4related disease. Clin Med (Lond). 2015;15(2):130-134.

13. Baptista B, Casian A, Gunawardena H, D'Cruz D, Rice CM. Neurological manifestations of IgG4-related disease. Curr Treat Options Neurol. 2017;19(4):14.

14. Katsura M, Mori H, Kunimatsu A, et al. Radiological features of IgG4-related disease in the head, neck, and brain. Neuroradiology. 2012;54(8):873-882.

15. Marinelli JP, Marvisi C, Vaglio A, et al. Manifestations of skull base IgG4-related disease: a multi-institutional study. Laryngoscope. 2020;130(11):2574-2580.

16. Okano A, Nakatomi $H$, Shibahara J, et al. Intracranial inflammatory pseudotumors associated with immunoglobulin G4-related disease mimicking multiple meningiomas: A case report and review of the literature. World Neurosurg. 2015;83(6):1181.e1-1181.e11814.

17. Cant $A$, Collard $B$. Oral medicine: Isolated unilateral hypoglossal nerve palsy. Br Dent J. 2018;225(2):95.

18. Moteki H, Yasuo M, Hamano $H$, Uehara T, Usami S. IgG4-related chronic rhinosinusitis: a new clinical entity of nasal disease. Acta Otolaryngol. 2011;131(5):518-526.

19. Gao Y, Zheng M, Cui L, et al. IgG4-related disease: association between chronic rhino-sinusitis and systemic symptoms. Eur Arch Otorhinolaryngol. 2018;275(8):2013-2019.

20. Cler SJ, Sharifai N, Baker B, et al. IgG4-related disease of the skull and skull base-a systematic review and report of two cases. World Neurosurg. 2021;150:179-196.e1.

\section{Disclosures}

The authors report no conflict of interest concerning the materials or methods used in this study or the findings specified in this paper.

\section{Author Contributions}

Conception and design: Suisa, Soustiel. Acquisition of data: all authors. Analysis and interpretation of data: Suisa. Drafting the article: Suisa, Grober. Critically revising the article: Soustiel. Reviewed submitted version of manuscript: Soustiel, Grober. Approved the final version of the manuscript on behalf of all authors: Suisa. Administrative/technical/ material support: Suisa. Study supervision: Soustiel.

\section{Correspondence}

Haggai Suisa: Galilee Medical Center, Nahariya, Israel. haggais@gmc. gov.il. 